\title{
Resúmenes del VI Encuentro Nacional de Medicina del Dormir*
}

\section{Comparación del cuestionario mexicano con el cuestionario Berlín para detección de SAOS en población mexicana ${ }^{\ddagger}$}

Romero-López Z, , Camacho-Ortuño L,§ Ochoa-Vázquez MD,§ Bazán-Ávila $L, \S$ Gracia-Ramos AE, Mata-Marín JA ${ }^{\S}$

\footnotetext{
‡Trabajo ganador del Premio a la Investigación

§IMSS, Centro Médico Nacional La Raza
}

Introducción: E1 SAOS es un problema relevante de salud pública. Aumenta la mortalidad hasta 4 veces cuando no es tratado, de ahí la importancia de lograr un diagnóstico oportuno. Los cuestionarios son una herramienta importante, y detectan eficazmente a los pacientes con alto riesgo. El cuestionario mexicano (CM) es el único diseñado específicamente para población mexicana, pero su empleo no se ha difundido aún. Hipótesis: El CM tiene mayor sensibilidad y especificidad que el cuestionario de Berlín para la detección de pacientes con SAOS en población mexicana. Objetivo: Comparar la sensibilidad y especificidad del cuestionario mexicano con las del cuestionario del Berlín para la detección de SAOS en población mexicana. Ma-

\footnotetext{
* La transcripción de los resúmenes es responsabilidad de la Academia Mexicana de Medicina del Dormir, así como el orden en que aparecen.
}

terial y métodos: Estudio transversal, se incluyeron 308 pacientes referidos a la Clínica de Sueño. Se realizó una historia clínica completa junto con el cuestionario de Berlín, posteriormente se aplicó el cuestionario mexicano y se realizó PSG nocturna. Se calculó el odds ratio (OR) a cada pregunta del $\mathrm{CM}$, y las que tuvieron un valor menor a 1.5 fueron eliminadas, quedándonos con una versión modificada del mismo (CMm). Se determinó el punto de corte a partir del cual representa un riesgo alto para SAOS a través de curvas ROC. Se comparó la sensibilidad, especificidad, valor predictivo positivo y valor predictivo negativo de ambos cuestionarios, y finalmente se compararon mediante curvas ROC. Resultados: Ocho preguntas tuvieron OR > 1.5 para SAOS; mediante curvas ROC se encontró que con 5 positivas existe riesgo alto para SAOS. La sensibilidad y especificidad fueron 75 y $100 \%$ para el CMm, y 90 y $25 \%$ para el cuestionario Berlín, para un IAH $>5$. En el análisis mediante curvas ROC, el área bajo la curva fue 0.87 en CMm vs. 0.57 en Berlín. Conclusiones: El CMm es una herramienta válida para detectar pacientes con alto riesgo de SAOS en nuestra población.

\section{Calidad del sueño en atletas de los XXII Juegos Centroamericanos y del Caribe, Veracruz 2014}

\section{Tlatoa-Ramírez HM, Ocaña-Servín HL, Márquez-López ML, Armenta- Velázquez JL, Tenorio-Chousal J, Jiménez-Colín PY}

Centro de Medicina de la Actividad Física y el Deporte Facultad de Medicina, Universidad Autónoma del Estado de México

Introducción: El sueño como proceso dinámico estrictamente regulado, disminuye la actividad motora voluntaria, presenta un ritmo circadiano que, de alterarse, se afectan los ritmos normales de recuperación en el sueño. Hipótesis: La tercera parte de los atletas encuestados presentará deterioro en su calidad de sueño. Objetivo: Evaluar la calidad de sueño en los atletas de los Juegos Centroamericanos y del Caribe, Veracruz, 2014. Material y métodos: El estudio se realizó en una muestra no probabilística con un diseño transversal. Para la evaluación del sueño se utilizó el cuestionario: índice de calidad del sueño de Pittsburgh. El estudio fue previamente revisado y aprobado por el Comité de Ética del Centro de Medicina de la Actividad Física y el Deporte (CEMAFyD). Resultados: Se realizaron 312 encuestas en atletas que participaron en los XXII Juegos Centroamericanos y del Caribe, la edad promedio fue de 24.0 \pm 5.3 años para las mujeres y de $25.4 \pm$ 6.3 años para los hombres $(p=0.11)$. Se obtuvo una mayor frecuencia para una buena calidad de sueño $(\mathrm{n}=176) v s$. una mala calidad de sueño $(n=136)$ con una diferencia estadística significativa entre ambas frecuencias $(\mathrm{p}=0.02)$ No hay asociación entre la calidad de sueño y el género de los atletas, sin embargo, al parecer existe una relación entre el tipo de deporte individual o de conjunto y la calidad del sueño $(p=0.01)$. Conclusiones: El 44\% de los atletas presentó mala calidad de sueño, dato de preocupación para una población con actividad física constante.

\section{Comorbilidades, calidad de sueño y calidad de vida}




\section{en pacientes con cáncer pulmonar localmente avanzado}

\begin{abstract}
Torre-Bouscoulet L, ${ }^{*, \neq}$ Arrieta-Rodríguez O,\$ Pérez-Padilla JR, ,*, Guzmán Barragán $S A$,* Reyes-Zúñiga $M M,{ }^{*, \neq}$ Martínez-Briseño $D^{*}$
\end{abstract}

*INER Ismael Cosío Villegas; ${ }^{\ddagger}$ Academia Mexicana de Medicina del Dormir; §INCan

Antecedentes: Los pacientes con cáncer pulmonar presentan condiciones médicas y comorbilidades que disminuyen la calidad de sueño y la calidad de vida y que pueden afectar la respuesta al tratamiento oncológico así como el pronóstico. Objetivo: El propósito de este estudio es investigar, en pacientes con cáncer pulmonar localmente avanzado, la prevalencia de condiciones médicas y comorbilidades que podrían ser revertidas antes del tratamiento oncológico. Estos factores podrían afectar la calidad de sueño y la calidad de vida. Métodos: Diseño del estudio: evaluación inicial de pacientes consecutivos con diagnóstico de cáncer pulmonar de células no pequeñas etapas IIIA y IIIB. Para investigar las comorbilidades se utilizó el cuestionario PLATINO (www.platino-alat.org/docs/ cuestionario_platino_mexico.pdf). Se aplicaron la escala de disnea (MRC), el índice de calidad del sueño de Pittsburg (PSQI) y la escala de calidad de vida de EuroQL. Las pruebas de función pulmonar fueron: espirometría, capacidad de difusión pulmonar de monóxido de carbono (DLCO) y gasometría arterial (ATS/ERS 2005). Las escalas de PSQI y EuroQL se analizaron de acuerdo con los criterios de los autores. Resultados: Se reclutaron 46 pacientes; (a) $32.6 \%$ de los pacientes refirieron tener una sola comorbilidad y $13.1 \%$ refirieron disnea; (b) la prevalencia de EPOC fue de $39.1 \%$ y $16.7 \%$ mostraron hipoxemia; (c) $50 \%$ reportaron una pobre calidad de sueño; (d) el promedio del puntaje de calidad de vida fue alto $(0.848[\mathrm{EE} \pm 0.185])$; $45.7 \%$ refirieron dolor y $19.6 \%$ síntomas de ansiedad y depresión; (e) pobre calidad del sueño se asoció significativamente a dolor y síntomas de ansiedad y depresión. Conclusión: Existen comorbilidades y condiciones médicas en pacientes con cáncer pulmonar localmente avanzado que podrían ser tratadas antes de iniciar el tratamiento con radio y quimioterapia. Uno de cada dos pacientes con cáncer pulmonar tiene pobre calidad de sueño asociado, principalmente, a dolor.

\section{Enfermedad residual en niños tratados con adenoamigdalectomía por apnea obstructiva del sueño grave: Resultados preliminares}

Taniyama-López ON, ${ }^{*}$ JiménezRodríguez J, * Reyes-Zúñiga MM, ,; Carrillo-Alduenda JL, ,; TorreBouscoulet $L, * ; \neq$ Torres-Fraga MG*,;

*INER Ismael Cosío Villegas; ${ }^{\ddagger}$ Academia Mexicana de Medicina del Dormir

Introducción: La apnea obstructiva de sueño es el trastorno respiratorio del sueño más frecuente en la población infantil. Objetivo: Explorar si existe enfermedad residual y cuál es la frecuencia de ésta en niños tratados con adenoamigdalectomía por apnea obstructiva del sueño (AOS) grave. Métodos: Estudio retrospectivo pacientes con AOS grave operados de adenoamigdalectomía en el INER. La enfermedad residual fue valorada mediante poligrafía un año después de la cirugía. Resultados: Datos de 35 pacientes, 21 hombres, edad 5.14 años. El IER fue 38.7. El perfil de uso de APAP mostró que el promedio de horas de uso por noche fue 4.9, y percentila 95 de presión $9.8 \mathrm{cmH}_{2} \mathrm{O}$. El IER posoperatorio fue de 8.6. $\mathrm{La} \mathrm{SaO}_{2}$ promedio 93\%, $\mathrm{SaO}_{2}$ mínima $79 \%$; $2.3 \%$ del tiempo de sueño la $\mathrm{SaO}_{2}$ es < 90\%. Conclusiones: La adenoamigdalectomía reduce significativamente el IER, sin embargo, persiste enfermedad residual, que amerita seguimiento multidisciplinario estrecho.

Consumo de oxígeno y pruebas de función respiratoria en pacientes con insuficiencia cardíaca

\section{grave más trastorno respiratorio del dormir}

\author{
Herrera-Flores $\mathrm{EH}^{*}{ }^{*}$ Carrillo- \\ Alduenda JL, ,; Torres-Fraga MG, *, \\ García-Polo ML, ${ }^{* \neq}$ Cigarroa-López $A,{ }^{\S}$ \\ Castorena-Maldonado $A R^{\star, \neq}$
}

*INER Ismael Cosío Villegas; ${ }^{\ddagger}$ Academia Mexicana de Medicina del Dormir; ${ }^{\S}$ Hospital de Cardiología del CMN SXXI

Introducción: La prevalencia de trastornos respiratorios del dormir (TRD) en insuficiencia cardíaca (IC) es del $52 \%$ y su presencia se asocia a mayor mortalidad y peor pronóstico. Los pacientes con IC grave tienen disminución del consumo de oxígeno $\left(\mathrm{VO}_{2}\right)$ máximo y esto se asocia a mayor mortalidad. No existen datos disponibles sobre alteraciones en $\mathrm{VO}_{2} \mathrm{ni}$ en pruebas de función respiratoria (PFR) en pacientes con IC grave más TRD. Objetivo: Comparar los valores del $\mathrm{VO}_{2}$ y PFR entre pacientes con IC grave más apnea obstructiva del sueño (AOS) y pacientes con IC grave más respiración de Cheyne Stokes (RCS). Material y métodos: Estudio de diseño descriptivo, transversal, en una cohorte del Hospital de Cardiología del Centro Médico Nacional Siglo XXI de pacientes con IC grave (FEVI < 35\%), en tratamiento óptimo al menos durante el mes previo y en protocolo de trasplante cardíaco, a los que se les realizó polisomnografía nocturna, espirometría, pletismografía corporal, capacidad de difusión de monóxido de carbono en respiración única, gasometría arterial (GA) basal y prueba de ejercicio cardiopulmonar (PECP). Resultados: Se evaluaron 70 sujetos, la edad media fue $46.8 \pm 13.2$ años, 58 fueron de sexo masculino $(82.9 \%)$. Se encontró 5 pacientes sin trastorno respiratorio del dormir, 22 con AOS, 38 con RCS y 5 con apnea central sin RCS. Los pacientes tenían características clínicas y ecocardiográficas similares excepto que el IMC fue mayor en pacientes con AOS $(29.7 \pm 65.3 \mathrm{vs}$. $25.7 \pm 3.6 ; \mathrm{p}=0.004)$. Conclusiones: La capacidad inspiratoria y el consumo de oxígeno máximo son menores en los pacientes con IC grave más RCS en comparación con aquellos que tienen IC grave más AOS. Los hallazgos probablemente 
sean secundarios a congestión pulmonar y menor función cardíaca.

\section{Efecto de la vacuna AH1N1 sobre el patrón de sueño de ratas restringidas de SMOR}

\author{
Hernández-Ortega SI," Rojas- \\ Zamorano JA, * Velázquez-Moctezuma \\ J,‡ Domínguez-Salazar E,‡ Jiménez- \\ Correa $U, \S$ Santana-Miranda $R^{\S}$
}

${ }^{*}$ Facultad de Estudios Superiores Zaragoza, UNAM; ¥Área de Neurociencias, Departamento de Biología de la Reproducción, CBS, UAM-Iztapalapa; §Clínica de Trastornos del Sueño, Facultad de Medicina, UNAM

En la primavera de 2009, una nueva cepa de la gripe AH1N1 apareció en México afectando principalmente adultos jóvenes con una tasa de letalidad de 0.4\%. En 2010 en Finlandia y Suecia, se reportaron casos de narcolepsia en la infancia después de aplicar la vacuna contra dicha cepa. Es posible que algún componente de la vacuna afecte al SNC, provocando un efecto sobre los mecanismos reguladores del ciclo vigilia-sueño. Reportes previos han evidenciado la permeabilidad de la barrera hematoencefálica (BHE) por la restricción de SMOR (RSMOR). El objetivo de este trabajo es establecer si hay una relación entre la aplicación de la vacunas contra la influenza AH1N1, y el desarrollo de alteraciones en el ciclo sueño-vigilia en ratas jóvenes cuya BHE ha sido vulnerada mediante la RSMOR. Hipótesis: La vacunación de AH1N1 a sujetos experimentales sometidos a RSMOR, causará alteración del patrón de sueño. Método: Se emplearon 24 ratas macho jóvenes de la cepa Wistar. Se les implantó un dispositivo supracraneal para registro convencional de sueño. Se les restringió de SMOR por el método de plataformas múltiples y al décimo día fueron inmunizadas, continuando con la restricción por cinco días más. 20 después se registraron polisomnográficamente por 8 horas durante el período obscuro. Resultados: $\mathrm{Se}$ registró un aumento en el tiempo total de sueño y de SMOR con respecto a los controles. Conclusiones: La aplicación de la vacuna es capaz de alterar el ciclo sueño-vigilia ante condiciones que vulneran la permeabilidad de la barrera hematoencefálica.

\section{Historia de tratamiento en pacientes con insomnio crónico}

\section{Quiroz-Casian L, ${ }^{*}$ Alfaro-Ávila EA,‡ Jiménez-Genchi $A$, * Martínez-} Fernández $A^{*}$

*Instituto Nacional de Psiquiatría Ramón de la Fuente; ¥Universidad Autónoma de Nayarit

Introducción: En México se presume una alta prevalencia del uso de terapias alternativas y automedicación, pero es escasa la información sobre el uso de estos tratamiento entre la población con insomnio. Hipótesis: Los pacientes con insomnio crónico recurren con elevada frecuencia a la herbolaria para reducir sus problemas con el sueño. Objetivo: Describir el tratamiento que practican los pacientes con insomnio antes de acudir a una clínica de sueño. Material y métodos: Se seleccionaron pacientes mayores de 18 años con insomnio crónico que acudieron a la clínica de sueño; se diseñó un cuestionario para evaluar la historia de tratamiento, todos con consentimiento verbal. Resultados: $\mathrm{Se}$ incluyeron 49 pacientes, (edad 51.2 años; $73.4 \%$ mujeres). La duración del insomnio fue de 10.2 años. El $79.6 \%$ empleó algún tratamiento para el insomnio, sin mostrar diferencias en edad y género pero si en la duración del insomnio en comparación con los que no habían empleado tratamiento alguno. Acudieron con médicos generales $46.2 \%$, médicos especialistas $33.3 \%$, médicos homeópatas $23.1 \%$, médicos psiquiatras $25.7 \%$, psicólogo $10.3 \%$, conocidos $2.6 \%$ y chaman $2.6 \%$. El tratamiento más empleado fue la herbolaria $76.9 \%$, seguido por las benzodiacepinas $43.6 \%$ y en menor porcentaje antidepresivos $15.4 \%$, otros remedios $12.8 \%$, ejercicio físico $12.8 \%$, la melatonina $10.3 \%$ y las vitaminas $5.1 \%$. El $65 \%$ solamente empleó 1 de los tratamientos antes descritos, principal- mente la herbolaria, el resto combinó 2 o más tratamientos siendo el más frecuente la herbolaria más benzodiacepina. De la herbolaria predominaron el azahar, valeriana, tila y pasiflora. Los sujetos dieron una calificación baja de 3.5/10 a los resultados con estos tratamientos. Conclusiones: Dentro de la historia del tratamiento los pacientes con insomnio acuden con médicos generales, después con médicos especialistas y homeópatas. Tres de cada 4 pacientes emplean sustancias de la herbolaria (azahar, valeriana, tila, pasiflora), destacando que la mejoría obtenida con estos tratamientos es pobre.

\section{Relación del ciclo vigilia- sueño sobre los hábitos alimentarios en obesos}

\section{Leija-Alva G, ${ }^{*}$ Orea-Tejeda $A^{\ddagger}$}

*Instituto Politécnico Nacional; ‡INER Ismael Cosío Villegas

Introducción: Los seres vivos se han adaptado a los movimientos de rotación y traslación del planeta y específicamente a las oscilaciones de iluminación y temperatura, han modificado la hora de dormir y despertar así como la cantidad de alimentos que ingiere. Como consecuencia han incrementado enfermedades como la obesidad y todas sus comorbilidades. En algunos casos estos problemas se relacionan con los cambios metabólicos relacionados con los ciclos vigilia-sueño. Objetivo: Identificar la relación entre los ciclos vigilia-sueño sobre los hábitos. Métodos: A un grupo de 45 personas obesas se les realizaron mediciones antropométricas y llenaron un diario de sueño y el cuestionario de sobreingesta alimentaria (O.Q.). Resultados: El 32\% tuvo un despertar a la semana. El $60 \%$ tuvo una sensación de algo renovado y el $32 \%$ renovado. El 16\% tuvo situaciones emocionales perturbadoras y otro $16 \%$ malestares físicos perturbadores de sueño (16\%). Previo a dormir el $60 \%$ vio televisión. La hora promedio de dormir y levantarse fue las 23:00 y 7 a.m. respectivamente. El número de horas de sueño fue 7 horas, con una latencia hasta lograrlo de $30 \mathrm{~min}$. Del cuestionario de 
ingesta alimentaria, la subingesta y afectividad tuvieron una correlación negativa significativa con el total de las horas de sueño (a menor cantidad de sueño, mayor ingesta alimentaria, así como del estado de ánimo negativo. Conclusiones: La obesidad como una enfermedad multifactorial requiere de la intervención de diversos especialistas, para prevenir, tratar y controlar este problema, que debía incluir otras disciplinas como la cronobiología, ciencia que nos recuerda que somos parte de la naturaleza y que nuestro funcionamiento bioquímico, fisiológico, comportamental y cognitivo, está regulado por relojes internos que están en estrecha relación con los ciclos de luz y sombra.

\section{EMTr de $5 \mathrm{~Hz}$ en CPFDL izquierda sobre las alteraciones del sueño en adultos con depresión}

\section{Carrillo-Sulub AM,* Moo-Estrella JA, * González-Olvera $\mathrm{J}^{\ddagger}$}

*Universidad Autónoma de Yucatán, Facultad
de Psicología; 'Instituto Nacional de Psiquiatría Ramón de la Fuente Muñiz

Introducción: La estimulación magnética transcraneal (EMTr) es una técnica aprobada para la depresión, pero se desconoce sus efectos sobre las alteraciones del sueño, considerado uno de los síntomas resistentes en el tratamiento del trastorno depresión mayor (TDM). Nuestro objetivo fue conocer el efecto de la EMTr a $5 \mathrm{~Hz}$ en la corteza prefrontal dorsolateral izquierda (CPFDLi) sobre las alteraciones del sueño en adultos con TDM. Material y métodos: Participaron 9 adultos con promedio de edad de 49.66 ( \pm 15.75$)$ años y el $78 \%$ mujeres, con diagnóstico de TDM. Se les aplicó el índice de calidad de sueño de Pittsburg (PQSI), el índice de gravedad de insomnio (ISI), la escala Hamilton de depresión y se les colocó el actígrafo MicroMini-Motionlogger (24/h durante siete días), antes y después del tratamiento (15 sesiones de 1,500 pulsos a 5 $\mathrm{Hz}$, durante 3 semanas). Resultados: Se encontró diferencia significativa en los síntomas de depresión de Hamilton (M $=28.66 \pm 5$ vs. $\mathrm{M}=14.11 \pm 7.45)$, pero no en el PQSI $(\mathrm{M}=9.77 \pm 3.70$ vs. $\mathrm{M}=$ $11.11 \pm 4.37)$, el ISI $(M=15.77 \pm 5.35$ vs. $\mathrm{M}=12.00 \pm 5.72$ ) y en la eficiencia, la latencia, los despertares y el índice de activación durante el sueño con actigrafía ( $p$ > .05). Conclusiones: La EMTr a $5 \mathrm{~Hz}$ en CPFDLi disminuye los síntomas de la depresión, pero no las alteraciones del sueño. Protocolos de EMTr específicos para las alteraciones del sueño, podrían acompañar el tratamiento para la depresión en futuras investigaciones.

\section{Hallazgos \\ electrofisiológicos en mujeres con síndrome de piernas inquietas comparado con sujetos control}

\section{Martínez-Fernández A, Jiménez- Genchi $A$}

Instituto Nacional de Psiquiatría Ramón de la Fuente Muñiz

Introducción: El síndrome de piernas inquietas (SPI) es un trastorno motosensorial, su diagnóstico es clínico y existen diferentes posibles mecanismos fisiopatológicos. La sintomatología que produce prolonga la latencia de sueño. La evaluación electrofisiológica no es un procedimiento de rutina para diagnóstico. La evaluación de la velocidad de conducción nerviosa (VCN) en conjunto con los reflejos $\mathrm{H}$ y la onda $\mathrm{F}$ evalúan la integridad de las vías posiblemente relacionadas con el SPI. Los reportes previos muestran resultados variables. Hipótesis: Los pacientes con SPI primario presentan alteraciones en la evaluación neurofisiológica. Objetivo: Comparar las VCN, reflejos $\mathrm{H}$ y onda $\mathrm{F}$ en extremidades inferiores de mujeres que padecen de SPI primario, con mujeres sanas de edad similar. Material y métodos: Estudio transversal, comparativo, con una población de 19 sujetos, 10 casos con SPI y 9 controles del sexo femenino, reclutados en el Instituto Nacional de Psiquiatría Ramón de la Fuente Muñiz, bajo consen- timiento informado y aprobación del Comité de Ética. Se obtuvieron los valores de VCN de los nervios peroneo, tibial y sural sensitivo, de manera bilateral, y la onda F y el reflejo H. Se realizó análisis estadístico mediante prueba $\mathrm{T}(\mathrm{p}<0.05)$ comparando los grupos. Resultados: El promedio de edad para el grupo de casos fue de 46.6 y el grupo control de 49.22 (p $=0.641$ ). Las velocidades de conducción (segmento peroneo tobillo-tibia izquierda y nervio sural sensitivo derecho) resultaron con diferencias significativas, siendo mayor el retardo en la conducción en los casos en comparación con los controles. No se encontraron diferencias en otras variables. Conclusiones: El retraso en las VCN es indicativo de compromiso nervioso a nivel periférico en SPI primario. No se puede atribuir a una disfunción aislada. Los datos encontrados no se contraponen a la posibilidad de diferentes tipos de SPI, diferentes mecanismos fisiopatológicos o la combinación de éstos.

\section{Dispositivo mexicano de avance mandibular (DAMm) en el control del SAOS}

\section{García-Campos EP,,; Galicia-Polo $M L^{\star \star \&, \|}$}

\footnotetext{
*Clínica de Trastornos del Sueño, UNAM Hospital General de México; \#Instituto Mexicano de Medicina Integral del Sueño (IMMIS); \&INER Ismael Cosío Villegas; "Academia Mexicana de Medicina del Dormir
}

Antecedentes: Debido a la prevalencia cada vez mayor de la presencia de ronquido y síndrome de apnea obstructiva del sueño no sólo en nuestra población sino a nivel mundial, ha motivado al interés no sólo por el especialista de la salud sino también del mismo paciente a la búsqueda de diversas alternativas terapéuticas en el control del mismo, siendo el uso de los dispositivos orales una de las alternativas que ha cobrado gran demanda en los últimos años. Sin embargo, a pesar de que existen a nivel mundial múltiples investigaciones sobre la eficacia del mismo, debido a la mala selección de los casos, a la comerciali- 
zación e incluso al mal manejo por la falta de seguimiento multidisciplinario así como también de un inadecuado entrenamiento en medicina del sueño, ha generado en muchos casos el fracaso y/o abandono del tratamiento, siendo que esta alternativa al ser bien seleccionado tanto el caso clínico como el tipo de dispositivo de acuerdo con cada paciente puede ser una excelente alternativa terapéutica en pacientes diagnosticados con ronquido habitual hasta casos de SAOS severa. Por lo que proponemos, en esta investigación, el manejo multidisciplinario de esta alternativa realizada en México, mediante el uso de un dispositivo de avance mandibular (DAMm), que a diferencia de los dispositivos utilizados actualmente en nuestro país, permita mayor comodidad para el paciente de acuerdo con sus propiedades físicas, al ser realizado individualmente, logrando con ello mayor tolerancia de uso durante la noche, además de brindar eficacia terapéutica al reducir los ronquidos y las apneas nocturnas en más de un $70 \%$, mediante un manejo multidisciplinario adecuado tanto en los criterios de evaluación clínica tanto para la selección de los casos y del tipo de dispositivo, así como en el seguimiento conjunto mediante métodos como la poligrafía nocturna y métodos digitales de análisis de vía aérea, procurando con ello alcanzar un alto beneficio terapéutico y económico. Objetivo: Realizar y evaluar el uso de un DAM (m) fabricado individualmente mediante diversas pruebas clínicas y poligráficas en 60 pacientes con SAOS manejados en forma multidisciplinaria dentro de la Clínica de Sueño UNAM. Metodología: Selección de 60 casos diagnosticados con SAOS mediante polisomnografía o poligrafía nocturna, que de acuerdo con las pruebas clínicas multidisciplinarias cumplan con los criterios de inclusión médicos y odontológicos para la colocación de un DAM. El seguimiento clínico se realizó cada 15 días y tres meses posteriores a su colocación, se evaluó la eficacia del DAM mediante poligrafía nocturna. Análisis estadístico realizado mediante $\mathrm{T}$ de Student para comparar medias de valores respiratorios entre los estudios pre y postratamiento. Resultados: Los resultados obtenidos muestran cambios significativos con el uso de DAM (IC:95\%-p < .05): Reducción en el IAH, índice de ronquidos, aumento de $\mathrm{SaO}_{2}$, escala Epworth así como también cambios significativos en la evaluación subjetiva del paciente y de la pareja. Conclusiones: El uso del DAM (m), utilizado en la presente investigación mostró que gracias a su mecanismo de acción al igual que los dispositivos utilizados actualmente puede reducir los eventos respiratorios durante la noche, sin embargo, lo que podemos destacar con el uso de este nuevo prototipo de DAM (m), que el paciente refiere mayor comodidad al ser realizado individualmente, lo que se reflejara con un mayor uso del mismo durante la noche, por otro lado es indispensable recalcar la importancia del manejo multidisciplinario con el uso de esta alternativa terapéutica tanto para la selección de casos como para la selección del dispositivo oral misma que debe ser realizada por personal dental con adecuado entrenamiento en medicina del sueño, así como también se destaca la importancia en el seguimiento del mismo mediante diversos métodos clínicos, lo cual permitirá una mayor beneficio terapéutico para el paciente.

\section{Efectividad de la terapia cognitivo-conductual en pacientes con insomnio crónico}

\section{Valencia-Carlo YE,} Jiménez-Guenchi $A$

Instituto Nacional de Psiquiatría Ramón de la Fuente Muñiz

Introducción: La persistencia del insomnio se asocia con un incremento en el riesgo de morbilidad médica, psiquiátrica, disminución del funcionamiento y deterioro de la calidad de vida. De las diferentes opciones terapéuticas disponibles, los agonistas de los receptores de benzodiazepina y la terapia cognitivoconductual (TCC) reportan los mejores resultados en la evidencia empírica 1, 2. Hipótesis: La terapia cognitivo-conductual es efectiva en el insomnio crónico. Objetivo: Determinar la efectividad de la terapia cognitivo-conductual en pacientes con insomnio crónico. Material y métodos: El diseño del estudio es de prueba pos-prueba, los participantes fueron pacientes mayores de edad, que asisten a consulta externa en la clínica de sueño del INPRF entre enero de 2012 y junio de 2015 y cumplieron con los criterios diagnósticos del DSM-IV TR para insomnio $(n=110)$. La aplicación de los siguientes instrumentos se hizo al inicio y final de la TCC: índice de severidad del insomnio (ISI), escala de somnolencia de Epworth, escala de prácticas de higiene del sueño (EPHS), cuestionario de ansiedad y preocupación sobre el sueño. Resultados: E1 95\% de los participantes obtuvieron puntajes mayores a 10 en la escala de somnolencia de Epworth, el $80 \%$ refirió que la severidad del insomnio fue moderada y grave. En la prueba de $\mathrm{T}$ de Student para muestras pareadas se encontró una diferencia estadísticamente significativa en los puntajes iniciales y finales en las cuatro escalas aplicadas: ISI (t: 5.9121, df: 66, p < 0.01), escala de Epworth (t: = 2.7310, df: 98, p < 0.007), EPHS (t: 6.9471, df: 107, p < 0.01), cuestionario de ansiedad y preocupación sobre el sueño (t: 5.7041, df: 104, p < 0.01). Conclusiones: Los resultados sugieren que la terapia cognitivo-conductual tiene un claro impacto positivo sobre el insomnio crónico. Se confirman los hallazgos reportados por investigaciones previas a nivel internacional.

\section{Apnea de la prematurez, estudio de caso \\ Domínguez-Sandoval G, *, Galicia-Polo $M L^{\S, \neq}$}

*UNAM, Facultad de Estudios Superiores, Iztacala; ${ }^{\ddagger}$ Academia Mexicana de Medicina del Dormir; §Clínica de Trastornos de Sueño, UNAM, Facultad de Medicina

La apnea central del prematuro es más frecuente cuanto menor es la edad gestacional al nacimiento, persiste hasta las 40 semanas de edad posconcepcional. La mayoría de las apneas centrales ocurren durante sueño MOR, etapa predominante en el RN pretérmino. Presentamos el caso de RN pretérmino (34 SDG) con 
Apgar 7/8, peso al nacer $1,500 \mathrm{~g}$ y talla $41 \mathrm{~cm}$, diagnosticado con polisomnografía nocturna con el Sx de apnea central del prematuro. Se realizaron 10 polisomnografías nocturnas, 6 durante los primeros 6 meses de vida posconcepcional, y 4 entre los 12 y 17 meses de edad. El IAH promedio fue de $61 / \mathrm{h}$ apneas centrales los primeros 6 meses, $\mathrm{SaO}_{2}$ promedio de $91 \%$ y $<90 \%=35 \%$ del TTS, la FC promedio de $111 \mathrm{lpm}$. La distribución del sueño fue la siguiente: $\mathrm{SQ}=43 \%, \mathrm{SA}=$ $53 \%$ y $\mathrm{SI}=4 \%$. En las polisomnografías realizadas entre 12-17 meses, se observó: IAH $=67 /$ h de predominio central, $\mathrm{SaO}_{2}$ promedio $=96 \%, \mathrm{SaO}_{2}<90 \%=10 \% \mathrm{del}$ TTS, FC promedio de 105 lpm. La distribución del sueño: $\mathrm{N} 1=3 \%, \mathrm{~N} 2=51 \%, \mathrm{~N} 3$ $=6 \%, \mathrm{R}=41 \%$. La persistencia de apneas centrales durante los primeros 6 meses de vida posnatal sin respuesta al tratamiento, sugiere evaluación polisomnográfica y seguimiento multidisciplinario que brinde tratamiento oportuno.

\section{Diferencias en el cuadro clínico entre pacientes preescolares y escolares con diagnóstico de síndrome de apnea obstructiva del sueño}

Torres-Valerio $\boldsymbol{R},{ }^{*}$ García-Villatoro MC, * García-Colín ER, * Torres-Fraga MG, ${ }^{*, \neq}$ Reyes-Zúñiga MM, ${ }^{*, \neq}$ delRazo-Rodríguez $R$, * Pérez-Padilla $R$, * Carrillo-Alduenda $\mathrm{JL}^{*, \neq}$

*INER Ismael Cosío Villegas; ${ }^{\ddagger}$ Academia Mexicana de Medicina del Dormir

Introducción: El síndrome de apnea obstructiva del sueño (SAOS) en edad pediátrica es muy frecuente, sin embargo, sus manifestaciones clínicas están poco reportadas y la edad de los pacientes podría influir sobre su cuadro clínico. El objetivo del estudio es comparar el cuadro clínico, comorbilidades y hallazgos poligráficos entre niños preescolares y escolares con SAOS. Material y métodos: Revisión de expedientes, se incluyeron niños de 2 a 12 años de edad, que acudieron a la Clínica de Trastornos Respiratorios del Sueño durante el 2014 con diagnóstico de SAOS confirmado por estudio de sueño. Resultados: Se incluyeron 244 pacientes (108 preescolares y 136 escolares). Los pacientes preescolares tuvieron mayor crecimiento amigdalino en comparación con los niños escolares. Las manifestaciones nocturnas fueron muy frecuentes y los síntomas diurnos fueron poco reportados en ambos grupos, excepto la intensidad del ronquido que fue mayor en el grupo de preescolares, no se encontraron diferencias en el cuadro clínico y en los antecedentes familiares. En SAOS fue más grave en los preescolares (RDI 26 vs. 19, TC90 19 vs. 13, $\mathrm{SpO}_{2}$ mínima 73 vs. 79, $\mathrm{p}<0.05)$. La frecuencia de enfermedadpor-reflujo-gastroesofágico (ERGE) fue mayor en preescolares mientras que los escolares presentaron mayor frecuencia de obesidad. Conclusiones: No existen diferencias significativas en el cuadro clínico y antecedentes familiares de los niños preescolares y escolares con SAOS. Los niños preescolares presentan índices más graves que los escolares. La comorbilidad más importante en escolares es la ERGE y en los escolares la obesidad.

\section{Manifestaciones clínicas y hallazgos polisomnográficos en niños con escoliosis}

García-Colín ER, ${ }^{*}$ Torres-Fraga

MG, ,* Reyes-Zúñiga MM,,; Baños-

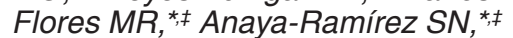

Pérez-Padilla $J R, * ;$ Carrillo-Alduenda $J L^{*, \neq}$

*INER Ismael Cosío Villegas; ‘Academia Mexicana de Medicina del Dormir

Introducción: Los trastornos respiratorios del sueño son frecuentes entre adultos con deformidad torácica; sin embargo, estos padecimientos están poco descritos en población pediátrica. Material y métodos: Revisión de expedientes, se incluyeron pacientes que acudieron a la Clínica de Trastornos Respiratorios del Sueño del Instituto Nacional de Enfermedades Respiratorias, México, del 1 enero 2010 al 1 enero 2015, menores a 18 años de edad, con diagnóstico de escoliosis. Todos los pacientes y sus familiares contestaron un cuestionario estandarizado de síntomas de sueño y se les realizó por lo menos una polisomnografía. Resultados: Se incluyeron 33 pacientes, 25 con escoliosis secundaria, edad $11 \pm 4.1$ años, con intercambio gaseoso diurno normal, sólo 6 tuvieron hipertrofia amigdalina. Los síntomas más frecuentemente reportados fueron: ronquido $(66 \%)$, despertares nocturnos (45\%), sueño no reparador $(45 \%)$ e irritabilidad diurna (43\%). Las comorbilidades más reportadas fueron: desnutrición (54\%) y antecedente de neumonía (54\%). En la polisomnografía se encontró una arquitectura de sueño normal, índice de alertamiento $10 \pm 6 \mathrm{~h}^{-1}$, índice de apnea hipopnea $6.2 \mathrm{~h}^{-1}$; el trastorno respiratorio del dormir más frecuente fue la hipoventilación durante el sueño $\left(\mathrm{CO}_{2}\right.$ promedio nocturno $40 \pm 9$ ), 17 pacientes (51\%) requirieron ventilación mecánica con un dispositivo de presión positiva nocturna. No se encontraron diferencias entre pacientes con escoliosis primaria y secundaria. Conclusiones: A pesar de no tener hipertrofia amigdalina, los niños con escoliosis manifiestan frecuentemente síntomas de TRD, el trastorno de sueño más importante fue la hipoventilación durante el sueño, y requieren ventilación mecánica nocturna a edades tempranas.

\section{Síndrome de Prader \\ Willi: Hallazgos polisomnográficos y estrategias ventilatorias}

\section{Cruz-Zorrilla G, ${ }^{*}$ Ramírez-Quiroz L, *, Domínguez-González HD, ,; Torres- Fraga MG, *; Reyes-Zúñiga MM, ,; Pérez-Padilla $J R,,{ }^{*}, \neq$ Carrillo-Alduenda $J L^{*, \neq}$}

*INER Ismael Cosío Villegas; ${ }^{\ddagger}$ Academia Mexicana de Medicina del Dormir

Introducción: El síndrome de Prader Willi (SPW) es un trastorno genético poco frecuente, que cursa con varios factores de riesgo para desarrollar trastornos respiratorios del dormir. El objetivo de este trabajo es describir los hallazgos polisomnográficos y la 
ventilación en pacientes con SPW. Material y métodos: Estudio retrospectivo, observacional, transversal. Se revisaron las PSG realizadas a pacientes con SPW entre 2005 y 2015. Resultados: Se encontraron 7 estudios correspondientes a 6 pacientes. Los pacientes con SPW presentan latencia acortada a sueño, 5 pacientes tuvieron SAOS, 1 de ellos asociado a hipoventilación. En 2 casos no se encontraron alteraciones. Los 5 paciente con SAOS requirieron tratamiento con un dispositivo de ventilación positiva. Conclusiones: Los trastornos respiratorios en pacientes con SPW son frecuentes y complejos. El dispositivo de presión positiva más utilizado fue Binivel S. La PSG es el estándar de referencia para el diagnóstico y titulación en pacientes con SPW.

\section{Actigrafía en pacientes con diabetes mellitus tipo 2, obesidad y apnea obstructiva del sueño}

\section{Chan-Chan ML, ${ }^{*}$ Rodríguez-Reyes $Y,{ }^{*}$ Huerta-Blancas $L,{ }^{*}$ Castorena- Maldonado $A^{* \neq}$}

*INER Ismael Cosío Villegas; ${ }^{\ddagger}$ Academia Mexicana de Medicina del Dormir

Objetivo: Describir y comparar parámetros de sueño (tiempo total de sueño, latencia a sueño, número de alertamientos, eficiencia al sueño) en pacientes con apnea del sueño, obesidad y diabetes mellitus tipo 2, obtenidos mediante la colocación de un actígrafo y a través de un cuestionario. Introducción: La actigrafía es un método que estima parámetros de sueño a través de un equipo portátil el cual registra el movimiento corporal. Material y métodos: 25 pacientes obesos (10 hombres, 15 mujeres con una edad promedio de 56 años \pm 8.53 años, IMC $35.2 \pm 2.16$ ) con diagnóstico de diabetes mellitus tipo 2 y apnea obstructiva del sueño moderada o grave (IRD promedio de $40.5+21.49$ ) sin tratamiento para la apnea del sueño. Se obtuvieron datos subjetivos relacionados con parámetros de sueño a través de un cuestionario y posteriormente se colocó un actígra- fo marca ActiGraph, durante 7 días. Resultados: Los pacientes reportaban dormir en promedio $8.08 \mathrm{~h} \pm 1.37 \mathrm{~h}$, el $48 \%$ dormía $<7$ horas, sin embargo, por actigrafía el tiempo total de sueño fue de $5.96 \mathrm{~h} \pm 1.17 \mathrm{~h}$ (p: 5.83), resultando en un $52 \%$ los pacientes que dormían menos de 7 horas. Asimismo, 11 pacientes (52\%) reportaron insomnio de inicio, sin poder ser comprobado por actigrafía ya que el promedio de latencia al sueño para los paciente fue de $1.14 \mathrm{~min} . \pm 0.73 \mathrm{~min}$., siendo la latencia a sueño más prolongada en duración de 2.57 min. Los pacientes reportaron 2.15 alertamientos por noche, encontrando por actigrafía 14.45 alertamientos \pm 5.24 por noche. Conclusiones: El uso de actígrafo como complemento del estudio en trastornos del sueño permite obtener datos objetivos referentes a los parámetros de sueño.

\section{Prevalencia de somnolencia excesiva diurna y apnea del sueño en una muestra de conductores de unidades y dependencias del Ejército Mexicano que acudieron al curso de adiestramiento en vehículos militares}

Castorena-Maldonado A, ${ }^{\text {S Sosa- }}$ Juárez $A,{ }^{\ddagger}$ Salazar-Soriano $A B, \$$ López-López JE, \& García-Licerio E, \& Chan-Chan $M,{ }^{*}$ Reyes-Zúñiga $M,{ }^{*, I}$ Carrillo-Alduenda $\mathrm{JL}^{*, \mathrm{Il}}$

*INER Ismael Cosío Villegas; ${ }^{\ddagger}$ Hospital Central Militar; §Programa Verano de la Ciencia Academia de Investigación Científicas; "Academia Mexicana de Medicina del Dormir

Introducción: La OMS y la junta para la seguridad en el transporte de Norteamérica han estimado que la somnolencia excesiva diurna (SED) representa aproximadamente el $30 \%$ de los accidentes a nivel global. Objetivo: Medir SED y apnea del sueño en una muestra de conductores de vehículos militares. Material y métodos: Estudio prospectivo, transversal, los síntomas del sueño fueron obtenidos por cuestionario, se uso la escala de Epworth, calidad de sueño de Pittsburg (ICSP) y la escala simplificada de cuello ajustada para calcular el riesgo de apnea del sueño (SACS). Resultados: Se entrevistaron a 122 militares masculinos con edad de $30 \pm 4.9$ años, el IMC fue $25 \pm 4.9 \mathrm{~kg} / \mathrm{m}^{2}$, la prevalencia de SED (Epworth $\geq 10$ ) fue $13 \%, 5 \%$ casi tuvo un accidente vehicular en servicio, el $21.5 \%$ refirieron haber cabeceado o dormido brevemente conduciendo entre una a dos veces por mes. La mayoría (73\%) suele tomar alguna medida conductual para mitigar el sueño como conversar con el copiloto o tomar refresco. La prevalencia de apnea del sueño (SACS $\geq$ 48) fue 6.5\%. En los sujetos con SED la calidad de vida subescala salud mental fue menor $(54.6 \pm 2.3$ vs. $58.6 \pm 4)$, y el ICSP fue mayor $(5.8 \pm 2.5$ vs. $3.7 \pm 1.5$, $\mathrm{p}=0.00)$. Conclusión: La prevalencia de SED y apnea del sueño es elevada en comparación con la población general, al ser una población relevante por la complejidad de los vehículos que conducen sería relevante realizar más estudios.

\section{Prueba de respuesta visual y toma de decisiones en residentes posterior a jornada laboral}

\section{Rodríguez-Reyes YG, ${ }^{*}$ Chan-Chan $M L$, * Huerta-Blancas $L,{ }^{*}$ Castorena- Maldonado $A^{*, \neq}$ \\ *INER Ismael Cosío Villegas; ${ }^{\ddagger}$ Academia Mexicana de Medicina del Dormir}

Antecedentes: Las horas de trabajo excesivas provocan alteraciones cognitivas y psicomotrices en los residentes. Objetivo: Evaluar el impacto de una guardia en la toma de decisiones de la vida cotidiana a través de una prueba psicológica, así como la velocidad de respuesta visual posterior a una guardia laboral. Metodología: Se invitó a participar a residentes de la Especialidad de Neumología en el INER que se encontraban bajo un esquema de guardias $\mathrm{ABCD}$. Se recabaron sus datos demográficos, hábitos de sueño, escala de somnolencia. Posterior a la realización de una guardia laboral se realizó prueba de 
respuesta visual (psychomotor vigilance task), y prueba de decisión (Iowa Gambling Task). Resultados: Se incluyeron a 19 residentes, 6 mujeres y 13 hombres, con una media de Epworth de 6, de los cuales se obtuvo un IGT con una media de raw score de 3 y una media de $\mathrm{t}$ score de 41.15 , con un tiempo de respuesta visual medio de 273.43 milisegundos con un tiempo mínimo de 197.799 milisegundos y un tiempo de respuesta lento promedio de 458.216 milisegundos. Conclusiones: La privación aguda de sueño tiene impacto principalmente en la toma de decisiones en los residentes, manteniéndose normales en la respuesta visual. $\triangle$ Correspondencia:

Dr. José Luis Carrillo-Alduenda Jefe de la Clínica de Sueño, Instituto Nacional de Enfermedades Respiratorias Ismael Cosío Villegas, Ciudad de México. Calzada de Tlalpan Núm. 4502, Colonia Sección XVI, 14080, México, D.F.

Correo electrónico:

jlcarrillo14@hotmail.com 